\title{
Valuation Perspectives and Decompositions for Variable Annuities with GMWB riders
}

\author{
Cody B. Hyndman ${ }^{\mathrm{a}, *}$, Menachem Wenger $\mathrm{a}^{\mathrm{a}, \mathrm{b}, * *}$ \\ ${ }^{a}$ Department of Mathematics and Statistics, Concordia University, 1455 De Maisonneuve \\ Blvd. W., Montréal, Québec, Canada H3G 1 M8 \\ ${ }^{b}$ The Guardian Life Insurance Company of America, New York, NY
}

\begin{abstract}
The guaranteed minimum withdrawal benefit (GMWB) rider, as an add on to a variable annuity (VA), guarantees the return of premiums in the form of periodic withdrawals while allowing policyholders to participate fully in any market gains. GMWB riders represent an embedded option on the account value with a fee structure that is different from typical financial derivatives. We consider fair pricing of the GMWB rider from a financial economic perspective. Particular focus is placed on the distinct perspectives of the insurer and policyholder and the unifying relationship. We extend a decomposition of the VA contract into components that reflect term-certain payments and embedded derivatives to the case where the policyholder has the option to surrender, or lapse, the contract early.
\end{abstract}

Keywords: Variable Annuity, GMWB, optimal stopping

\section{Introduction}

\subsection{Background}

Variable deferred annuities have two phases: the accumulation period and the annuitization period. During the accumulation period, premiums are deposited with the insurer and can be actively managed by the policyholder to achieve investment goals by allocating the funds to a selection of investment funds. The policyholder may choose to take partial withdrawals and/or surrender the contract, although the proceeds will likely be subject to contingent deferred sales charges (CDSC) and possible tax penalties depending on the age of the policyholder. Upon annuitization the policyholder cedes control over the funds and in return is guaranteed a periodic stream of payments, ranging from a

\footnotetext{
${ }^{*}$ Corresponding author

${ }^{* *}$ The views and opinions expressed in this paper are those of the individual author(s) and do not necessarily reflect the views of The Guardian Life Insurance Company of America.

Email addresses: cody.hyndman@concordia.ca (Cody B. Hyndman), menachem_wenger@glic.com (Menachem Wenger)
} 
fixed number of years up to guaranteed for life. This phase protects annuitants from longevity risk.

Riders are optional add-ons to variable annuities (VA), providing additional benefits in return for which an additional charge is subtracted annually from the account value $(\mathrm{AV})$. The first riders introduced to the VA market were death benefit riders: these guarantee a minimum death benefit to the beneficiaries if the policyholder dies during the accumulation period. Death benefits evolved from a simple return of premium to increasingly rich guarantees in the form of annual roll-ups and highest anniversary values. The next form of riders introduced were guaranteed living benefits (GLBs). The guaranteed minimum accumulation benefit riders (GMABs) guarantee a minimum account value at a specific date (i.e., 10 years from issue date), while the guaranteed minimum income benefit riders (GMIBs) guarantee a minimum annuitization amount by giving policyholders the choice between annuitizing a higher guarantee base at contractually specified annuitization rates or the current account value at the current annuitization rates.

Guaranteed minimum withdrawal benefit riders (GMWBs) were introduced in 2002 and guarantee the policyholder will recover at least the total premiums paid into the policy in the form of periodic withdrawals, subject to the annual withdrawals not exceeding a contractual percentage of the premiums. By allowing policyholders to remain in the accumulation phase and retain full control of their investments, policyholders benefit from the upside potential of equity investments while being protected from downside risk. GMWBs evolved into the guaranteed lifetime withdrawal benefit riders (GLWBs) which guarantee the annual maximal withdrawals for life. GMWB and GLWB riders represent embedded financial put options on the account values and techniques from mathematical finance have been used to value these contracts.

The fee structures of VA riders add complexity to pricing and risk management processes relative to the standard financial equity market derivatives. For a standard financial option a single upfront premium is charged which has no impact on the future random payoffs. No upfront fees are charged for GMWB riders but instead fees are deducted periodically from the AV to pay for the rider where the fees are proportional to the AV. The AV is influenced by the withdrawal behaviour of the policyholder and fee revenue stops in the event of death or surrender. As such there are multiple sources of uncertainty surrounding the fees. As well, an increase in the fee rate results in higher fee income but it also creates a drag on the AV, potentially causing it to reach zero faster which would result in earlier termination of fee revenues and increased rider guarantee payouts.

It is our belief that the GMWB and GLWB riders play a more prominent role in driving sales of VAs and their accompanying profits rather than as a source of direct profit for insurers. Shortly, we point to the consensus among the early papers that these riders were underpriced, supporting this hypothesis that they were only a means to increase VA sales. Indeed, reinsuring all or most of the risk was a popular risk management strategy for the initial GMWB products. Reinsurance premiums increased as reinsurers became more informed of the high 
risk embedded in these products. Around the time of the financial crisis in 2008 reinsurers stopped offering coverage altogether on GMWB and GLWB riders at which point the importance of internal dynamic hedging programs rose rapidly.

With this in mind, we consider pricing and hedging the GMWB product in a simplified framework consistent with the no-arbitrage principle from financial economics. GLB riders have grown increasingly complex in recent years. Added features range from periodic ratchets and annual roll-ups to specific one-time bonuses if certain criteria are met. These features were designed to both increase the product appeal and entice policyholders to delay withdrawals to the benefit of the insurer. It is evident that the GLWB riders have come to define the VA market. GMWB riders were the precursor to the GLWBs and as such, a mathematical analysis of the GMWB product is interesting in its own right, and can provide insights that may be extended to more complex products.

\subsection{Product Specifications and Notation}

An underlying VA contract plus a GMWB rider is issued at time $t=0$ to a policyholder of age $x$ and an initial premium $P$ is received. We assume no subsequent premiums. The premium is invested into a fund which perfectly tracks a risky asset $S=\left\{S_{t} ; t \geq 0\right\}$ with no basis risk. The rider fee rate $\alpha$ is applied to the account value $W=\left\{W_{t} ; t \geq 0\right\}$. Fees are deducted from the account value as long as the contract is in force and the account value is positive.

A guaranteed maximal withdrawal rate $g$ is contractually specified and the rider specifies that up to the amount $G:=g P$ can be withdrawn annually until $P$ is recovered, regardless of the evolution of $\left\{W_{t}\right\}$. If the account value hits zero, then the policyholder receives withdrawals at rate $G$ until the initial premium has been recovered. Policyholders always have the option of withdrawing any amount provided it does not exceed the remaining account value. If annual withdrawals exceed $G$ while the account value is still positive, then a surrender charge is applied to the withdrawals and a reset feature may reduce the guarantee value, e.g., the remaining portion of the initial premium not yet recovered. Policyholders also have the option of surrendering early (the terminology of lapses and surrenders are used interchangeably) and receiving the account value less a surrender charge. Any guarantee value is forfeited by surrendering.

Assuming a static withdrawal strategy where $G$ is withdrawn annually we set the maturity $T:=1 / g$ so that cumulative withdrawals at time $T$ equal $P$. At time $T$ the rider guarantee is worthless and the policyholder receives a terminal payoff of the remaining account value, if it is positive. This assumption translates over to a real-world trend of no annuitizations and is justified since a high proportion of VAs are not annuitized.

\subsection{Literature Review}

The initial paper on pricing and hedging GMWB products, by (Milevsky and Salisbury, 2006), employs continuous withdrawals and a standard geometric Brownian motion model for $\left\{S_{t}\right\}$ and considers two policyholder behaviour strategies. Under a static withdrawal strategy and no lapses the contract is decomposed into a 
term certain component and a Quanto Asian Put option. Numerical PDE methods are used to evaluate the ruin probabilities for $\left\{W_{t}\right\}$ and the contract value $V_{0}$. A dynamic behaviour strategy is considered where optimal withdrawals occur. This free boundary value problem is solved numerically for $V_{0}$. It is found that the optimal strategy reduces to withdrawing $G$ continuously unless $W_{t}$ exceeds a boundary value depending on the remaining guarantee balance of $P-G t$, in which case an arbitrarily large withdrawal rate is taken and the policyholder should lapse. (Milevsky and Salisbury, 2006) conclude that the GMWB riders in effect in 2004 were underpriced.

The optimal behaviour approach is formalized in (Dai et al., 2008) where a singular stochastic control problem is posed. Unlike in (Milevsky and Salisbury, 2006), time dependency and a complete description of the auxiliary conditions are included in this model. To facilitate numerical solutions for the HJB equations a penalty approximation formulation is solved using finite difference methods. Consistent with (Milevsky and Salisbury, 2006), numerical results provide support that the provision for optimal behaviour is quite valuable and insurers appeared to be underpricing GMWB riders. The optimal strategy consists of withdrawing at rate $G$ (continuously) except for in certain regions of the state space where an infinite withdrawal rate is optimal, which means to "withdraw an appropriate finite amount instantaneously making the equity value of the personal account and guarantee balance to fall to the level that it becomes optimal for him to withdraw $[G]$ " (Dai et al., 2008). However, (Dai et al., 2008) allow the policyholder the option of withdrawing any amount of the unrecovered initial premium, even if it exceeds the account value. In other words, if the account value is zero, the policyholder can elect to receive the remaining guarantee balance instantly subject to surrender charges rather than receive $G$ annually. The impact of this assumption is amplified by not including a reset feature in most of their work. The combination of this is the main cause of arriving at optimal strategies differing from (Milevskv and Salisbury, 2006).

(Chen and Forsyth, 2008) extend (Dai et al., 2008) to an impulse control problem representation where the control set allows for continuous withdrawal rates not exceeding $G$ and instantaneous finite withdrawals. This extension allows for modelling of more complex product features.

(Bauer et al., 2008) develop an extensive and comprehensive framework to price any of the common guarantees available with VAs, assuming that any policyholder events such as surrenders, withdrawals, or death occurs at the end of the year. Deterministic mortality is assumed. Monte-Carlo simulation is used to price the contracts assuming a deterministic behaviour strategy for the policyholders. To price the contracts assuming an optimal withdrawal strategy, a quasi-analytic integral solution is derived and an algorithm is developed by approximating the integrals using a multidimensional discretization approach via a finite mesh. Hence, only a finite subset of all possible strategies are considered. One drawback is that the valuation with optimal behaviour for a single contract could take excessive computation time.

Allowing for discrete withdrawals, (Bacinello et al., 2011) consider a number of guarantees under a more general financial model with stochastic interest rates 
and stochastic volatility in addition to stochastic mortality. In particular for GMWBs, a static behaviour strategy ( $G$ withdrawn annually and no lapses) is priced using standard Monte Carlo whereas an optimal lapse approach $(G$ withdrawn annually) is priced with a Least Squares Monte Carlo algorithm.

Upper and lower bounds on the price process for the GMWB are derived in (Peng et al., 2012) under stochastic interest rates and assuming a static continuous withdrawal strategy of $G$ per year with no lapses. (Peng et al., 2012) also present a tangential result about the relationship between the insured and insurer perspectives which we generalize to include early surrender.

Ignoring mortality and working with a static withdrawal assumption and no lapses, the primary focus of (Liu, 2010) is on developing semi-static hedging strategies under both a geometric Brownian motion model and a Heston stochastic volatility model for the underlying asset $\left\{S_{t}\right\}$. However, sufficient attention and detail is paid to pricing the GMWB rider assuming the insured takes constant withdrawals of $G / n$ at the end of each period where there are $n$ time steps per year. (Liu, 2010) observes that the contract (GMWB plus VA) can be decomposed into a term certain component and a floating strike Asian Call option on a modified process. Both a Monte Carlo approach and a moment-matching log-normal approximation method (based on Levy, 1992) are used to obtain results for increasing $n$.

\subsection{Overview}

The literature covers a wide range of theoretical and numerical approaches to modelling variable annuities with GMWB riders. The models surveyed differ most importantly in how they integrate model complexity and policyholder be-

haviour. Our approach begins with the model presented by (Milevsky and Salisbury, 2006) and establishes several new analytical results and relationships.

Based on the product specifications listed in Subsection 1.2, which shall be assumed throughout this paper, optimal withdrawal behaviour reduces to withdrawing at rate $G$ or lapsing. The rider guarantee represents an intangible amount. Once the account value is zero, this amount is accessible only through withdrawals at rate $G$, a product specification adopted by both (Milevskv and Salisburv, 2006) and (Bacinello et al., 2011). The work of (Dai et al., 2008) and (Chen and Forsyth, 2008) do not reflect this and therefore different results are obtained.

In Section 2 we motivate our work by reviewing the continuous-time model of (Milevsky and Salisbury, 2006). In Section 3 we formalize the relationship between the value processes for the GMWB rider from the point of view of both the insured and the insurer. We start with a restricted model which accounts for equity risk only. We provide our first results on the existence and uniqueness of a fair fee and a decomposition of the contract into the account value and the guarantee in the no lapse case. In Section 4 we extend the model to incorporate early surrenders, that is we incorporate policyholder behaviour risk, and subsequently generalize the decomposition of the value of the contract into the account value, the value of the guarantee under no-lapses, and the value of the option to lapse. Section 5 concludes and an appendix contains additional technical results and proofs. 


\section{Valuation of GMWBs in a Continuous-Time Framework}

We first consider the continuous model introduced by (Milevsky and Salisbury, 2006). This will motivate the developments in the following sections.

Let $(\Omega, \mathcal{F}, \mathbb{P})$ be a complete probability space where $\left\{B_{t}^{\prime}\right\}_{0 \leq t \leq T}$ is a one dimensional standard Brownian motion and $T<\infty$. Define $\mathcal{F}_{t}:=\sigma\left\{B_{s}^{\prime} ; 0 \leq\right.$ $s \leq t\}$, for all $t \in[0, T]$. Consider the financial market consisting of one risky asset and one riskless asset. The unit price of the risky asset $\left\{S_{t}^{x, u}\right\}_{u \leq t \leq T}$ follows the geometric Brownian motion process

$$
d S_{t}=\mu S_{t} d t+\sigma S_{t} d B_{t}^{\prime}, \quad t \geq u, \quad S_{u}=x .
$$

We assume a constant riskless rate $r$; therefore, the riskless asset is the money market account $M_{t}=e^{r t}$ for all $t \geq 0$.

Applying Girsanov's theorem for Brownian motion, $\left\{S_{t}^{x, u}\right\}_{u \leq t \leq T}$ follows the process:

$$
d S_{t}=r S_{t} d t+\sigma S_{t} d B_{t}, \quad t \geq u, \quad S_{u}=x .
$$

where $\left\{B_{t}\right\}$ is a standard Brownian motion under the unique risk neutral measure $\mathbb{Q}$ equivalent to $\mathbb{P}$. We work with the filtered probability space $\left(\Omega, \mathcal{F}_{T}, \mathbb{F}, \mathbb{Q}\right)$ where $\mathbb{F}=\left\{\mathcal{F}_{s}\right\}_{0 \leq s \leq T}$. The financial market is complete and arbitrage-free.

We formulate our initial assumptions as follows.

Assumption 1. We assume a static withdrawal strategy where the policyholder takes continuous withdrawals at a rate of $G:=g P$ per year. The maturity is $T:=\frac{1}{g}$ years. Early lapses are not permitted. We also assume $r>0$.

The account value process $\left\{W_{t}\right\}$ is reduced by the instantaneous rider fees $\alpha W_{t} d t$ and the instantaneous withdrawals $G d t$. By (2) the account value $W_{t}^{P, 0}$ follows the SDE

$$
d W_{t}=(r-\alpha) W_{t} d t+\sigma W_{t} d B_{t}-G d t, \quad 0 \leq t \leq T, \quad W_{0}=P .
$$

An additional constraint must account for the non-negativity of the account value. As stated in (Milevsky and Salisbury, 2006), it can be shown that $W$ satisfies

$$
W_{t}^{x, u}=\left(x e^{\left(r-\alpha-\frac{1}{2} \sigma^{2}\right)(t-u)+\sigma\left(B_{t}-B_{u}\right)}-G \int_{u}^{t} e^{\left(r-\alpha-\frac{1}{2} \sigma^{2}\right)(t-s)+\sigma\left(B_{t}-B_{s}\right)} d s\right)^{+}
$$

where $(w)^{+}=\max (0, w)$.

Considering $W_{t}^{P, 0}$, the initial premium $P$ can be factored out of (4) because $G=g P=P / T$. Let $\left\{Z_{t}\right\}$ denote the account value process under a no-withdrawal strategy beginning with $Z_{0}=1$. Then $Z_{t}$ follows the SDE

$$
d Z_{t}=(r-\alpha) Z_{t} d t+\sigma Z_{t} d B_{t}, \quad 0 \leq t \leq T, \quad Z_{0}=1,
$$

with the solution

$$
Z_{t}=e^{\left(r-\alpha-0.5 \sigma^{2}\right) t+\sigma B_{t}} .
$$


By (41) $W_{t}$ can be expressed in terms of $Z_{t}$ :

$$
W_{t}=\max \left[0, P Z_{t}-G \int_{0}^{t} \frac{Z_{t}}{Z_{s}} d s\right] .
$$

(Milevsky and Salisbury, 2006) use a slight variant of this expression involving the inverse of $Z$.

\section{Valuation Perspectives and Decompositions}

There are two perspectives from which to view the GMWB rider. A policyholder is likely to view the VA and rider as one combined instrument and would be interested in the total payments received over the duration of the contract. On the other hand, although the rider is embedded into the VA, the insurer might want to consider it as a separate instrument. Namely, the insurer is interested in mitigating and hedging the additional risk attributed to the rider.

\subsection{Policyholder Valuation Perspective}

Using standard actuarial notation we write the present value of a continuously paid term-certain annuity as

$$
\overline{\mathrm{a}}_{T \mathrm{~T}}=\int_{0}^{T} e^{-r s} d s=\frac{1-e^{-r T}}{r} .
$$

Denote by $V_{0}$ the value at $t=0$ for the complete contract (VA plus GMWB rider). The risk-neutral discounted value of the withdrawals and any terminal account value is

$$
V_{0}(P, \alpha, g)=E_{\mathbb{Q}}\left[\int_{0}^{T} G e^{-r s} d s+e^{-r T} W_{T}\right]=G \bar{a}_{T}+e^{-r T} E_{\mathbb{Q}}\left[W_{T}\right],
$$

as in (Milevsky and Salisbury, 2006). We write $V_{0}$ when the parameters are understood. Note that $V_{0}$ is an implicit function of the fee rate $\alpha$.

Let $\left\{V_{t}\right\}_{0 \leq t \leq T}$ denote the value process of the contract where $V_{t}$ considers only future cash flows occurring after time $t$, discounted to time $t$, conditional on the information $\mathcal{F}_{t}$. Then

$$
\begin{aligned}
& V_{t}=E_{\mathbb{Q}}\left[\int_{t}^{T} e^{-r(s-t)} G d s+e^{-r(T-t)} W_{T} \mid \mathcal{F}_{t}\right] \\
& =G \overline{\mathrm{a}} \frac{}{T-t \mid}+e^{-r(T-t)} E_{\mathbb{Q}}\left[W_{T}^{P, 0} \mid \mathcal{F}_{t}\right] .
\end{aligned}
$$

By the Markov property for $W_{t}$ we have $V_{t}=v\left(t, W_{t}\right)$, $\mathbb{Q}$-a.s., for all $t \in[0, T]$, where $v:[0, T] \times \mathbb{R}_{+} \mapsto \mathbb{R}_{+}$is given by

$$
v(t, x)=G \overline{\mathrm{a}} \frac{\overline{T-t}}{T}+e^{-r(T-t)} E_{\mathbb{Q}}\left[W_{T}^{x, t}\right] .
$$


Alternatively, $V_{0}$ can be decomposed into the sum of a term certain annuity component and either a Quanto Asian Put option on $Z^{-1}$ (see Milevsky and Salisbury, 2006) or an Asian Call (floating strike) option on $Z$ (see Liu, 2010). In either formulation the value function $v$ must be a function of both $Z_{t}$ and some functional $f\left(\left\{Z_{s} ; 0 \leq s \leq t\right\}\right)$ because only the joint process $\left\{Z_{u}, f\left(\left\{Z_{s} ; 0 \leq s \leq u\right\}\right)\right.$ is Markovian. Therefore we choose to continue working directly with (6). However, the alternative forms prove to be useful when we explore numerical implementations, a binomial model, and mortality diversification in companion papers (Hyndman and Wenger, 2013b,a)

Definition 2. A fair fee rate is a rate $\alpha^{\star} \geq 0$ such that

$$
V_{0}\left(P, \alpha^{\star}, g\right)=P .
$$

Equation (8) does not have a closed form solution and numerical methods must be used to find $\alpha^{\star}$. Since $P$ can be factored out of (4) it follows that $V_{0}(P, \alpha, g)=P V_{0}(1, \alpha, g)$ and from (8) it is immediate that $\alpha^{\star}$ is independent of $P$. A key question is the existence and uniqueness results of the fair fee rate. Before answering this question we first consider the insurer's valuation problem.

\subsection{Insurer Valuation Perspective}

The alternative viewpoint, applicable to the insurer, is to explicitly consider the embedded guarantee represented by the rider as a standalone product. Recall the trigger time defined by (Milevsky and Salisbury, 2006).

Definition 3. The trigger time, $\tau$, defined by the stopping time

$$
\tau:=\inf \left\{s \in[0, T] ; W_{s}^{P, 0}=0\right\},
$$

is the first hitting time of zero by the account value process. The convention $\inf (\emptyset)=\infty$ is adopted. If $\tau \leq T$ we say the option is triggered (or exercised) at trigger time $\tau$.

We have $W_{t}=0$ for all $t \geq \tau$. We define the respective non-decreasing sequences of stopping times $\left\{\tau_{t}\right\}_{t \in[0, T]}$ and $\left\{\bar{\tau}_{t}\right\}_{t \in[0, T]}$ as $\tau_{t}:=\tau \vee t$ and $\bar{\tau}_{t}:=$ $\tau_{t} \wedge T$, for all $t \in[0, T]$. Intuitively, if trigger occurred prior to $t$ then $\tau_{t}$ discards the information on the exact timing of the event. Further, since $\tau$ and $\tau_{t}$ could be infinite and the contract expires at time $T, \bar{\tau}_{t}$ is the minimum of $\tau_{t}$ and $T$.

For $0 \leq s \leq t \leq T$ and $A \subset[0, T]$, by the Markov property of $W_{t}$ we have

$$
\mathbb{Q}\left(\bar{\tau}_{t} \in A \mid \mathcal{F}_{s}\right)=F\left(s, t, A, W_{s}\right),
$$

$\mathbb{Q}$-a.s. where

$$
F(s, t, A, w):=\mathbb{Q}\left(\bar{\tau}_{t}^{w, s} \in A\right)
$$

and

$$
\bar{\tau}_{t}^{w, s}=\inf \left\{u \geq t ; W_{u}^{w, s}=0\right\} \wedge T .
$$

Let $U=\left\{U_{t} ; 0 \leq t \leq T\right\}$ denote the rider value process. At time $\bar{\tau}_{0}$ the rider guarantee entitles the policyholder to receive a term certain annuity for $T-\bar{\tau}_{0}$ 
years with an annual payment of $G$ and no uncertainty remains. Fee revenue is received up to time $\bar{\tau}_{0}$.

This motivates the following definition for $U$ which appears in (Peng et al., 2012). For $t \in[0, T]$ we define

$$
U_{t}:=E_{\mathbb{Q}}\left[e^{-r\left(\bar{\tau}_{t}-t\right)} G \overline{\mathrm{a}} \overline{T-\bar{\tau}_{t}}-\int_{t}^{\bar{\tau}_{t}} e^{-r(s-t)} \alpha W_{s}^{P, 0} d s \mid \mathcal{F}_{t}\right] .
$$

The value $U_{t}$ represents the remaining risk exposure to the insurer and is the risk-neutral expected discounted difference between future rider payouts and future fee revenues. By the Markov property for $\left\{W_{t}\right\}$ and (9) we have $U_{t}=$ $u\left(t, W_{t}\right), \mathbb{Q}$-a.s. for all $t \in[0, T]$, where $u:[0, T] \times \mathbb{R}_{+} \mapsto \mathbb{R}$ is given by

$$
u(t, x)=E_{\mathbb{Q}}\left[e^{-r\left(\bar{\tau}_{t}^{x, t}-t\right)} G \overline{\mathrm{a}} \frac{\bar{\tau}_{t}^{x, t}}{T-\int_{t}^{\bar{\tau}_{t}^{x, t}}} e^{-r(s-t)} \alpha W_{s}^{x, t} d s\right] .
$$

The boundary condition $u(t, 0)=G \overline{\mathrm{a}} \overline{T-t} \mid$ is implied in the above formulation.

\subsection{Analytic Results}

With the goal of arriving at an existence and uniqueness result for $\alpha^{\star}$, we first state two basic properties satisfied by $V_{0}$, the proofs of which can be found in the appendix.

Lemma 4. $V_{0}$, defined by (6) , is a strictly decreasing and continuous function of $\alpha$ for $\alpha \geq 0$.

Theorem 5. Under Assumption 1 there exists a unique $\alpha^{\star}$ satisfying

$$
V_{0}\left(P, \alpha^{\star}, g\right)=P \text {. }
$$

Remark. Assumption 1 imposed that $r>0$. Otherwise, the optimal solution $\alpha^{\star}$ must satisfy $W_{T}\left(\alpha^{\star}\right)=0, \mathbb{Q}$ a.s. and, by Lemma A2 of the Appendix, no solution exists.

The next result unifies the insured and insurer perspectives and was first presented in (Peng et al., 2012) for the case $t=0$ with stochastic interest rates. In Section 4 we extend this result to the more general case of surrenders and a complete proof will be presented at that time.

Proposition 6. For any $\alpha \geq 0$

$$
v(t, w)=u(t, w)+w
$$

for all $t \in[0, T]$ and $w>0$. That is, $V_{t}=U_{t}+W_{t}, \mathbb{Q}$ a.s.

Remark. By definition of the fair fee rate $\alpha^{\star}$ we have $U_{0}\left(P, \alpha^{\star}, g\right)=0$ as a result of Proposition 6. From Lemma 4 we have $V_{0}<P$ and $U_{0}<0$ for all $\alpha>\alpha^{\star}$. Likewise, $V_{0}>P$ and $U_{0}>0$ for all $\alpha<\alpha^{\star}$. For any $t$, we say the contract is in the money (ITM) if $V_{t}>W_{t}$ and $U_{t}>0$. Similarly, it is out of the money (OTM) if $V_{t}<W_{t}$ and $U_{t}<0$. It is at the money (ATM) if $V_{t}=W_{t}$ and $U_{t}=0$. 
Remark. In Subsection 1.1 we briefly discussed the fund drag created by an increase in the rider fee rate. The strictly decreasing property of $V_{0}$ and Proposition 6 imply that $U_{0}=V_{0}-P$ is a strictly decreasing function of $\alpha$. Thus any increase in expected revenue from an increase in $\alpha$ will always exceed any increase in expected rider payouts.

\section{Optimal Stopping and Surrenders}

We next extend the model to allow the policyholder to surrender, or lapse, the policy prior to time $T$. Although a policyholder may surrender for a number of reasons, for instance due to an emergency cash crisis, rational behaviour in an economic sense is assumed. Early surrenders occur only if the proceeds exceed the risk-neutral value of keeping the contract in-force.

Upon surrender the policyholder closes out the contract by withdrawing the current account value. The cash proceeds are reduced by a surrender charge on any amount exceeding the annual maximal permitted withdrawal amount specified in the rider contract. Typically, contract provisions include contingent deferred sales charge (CDSC) schedules specifying surrender charges as a function of the duration since issue year. An example is an 8-year schedule with a charge of $8 \%$ in year 1 and decreasing by $1 \%$ each year, followed by no surrender charges after year 9 .

To describe the CDSC schedule let $k:[0, T] \mapsto[0,1]$ be a deterministic nonincreasing piecewise constant RCLL (right continuous with left limits) function with possible discontinuities at integer time values. Our results hold for any non-increasing function taking values in $[0,1]$ but we select a function that is an accurate representation of CDSC schedules for products sold in the insurance marketplace. For a policy issued at time zero, $k_{s}$ is the surrender charge applicable at time $s$. The no-lapse model is easily recovered by setting $k_{s}=1$ for all $s \in[0, T)$ and $k_{T}=0$ in which case the opportunity to surrender early is worthless. Similarly, we could model a contract which only allows surrenders once a specific duration $t_{1}$ is reached, by setting $k_{s}=1$ for $s \in\left[0, t_{1}\right)$ and $k_{s}<1$ for $s \in\left[t_{1}, T\right]$. However the more common case has $k_{s}<1$ for all $s \in[0, T]$. Further, we assume $k_{T}=0$ to allow comparison to the no-lapse model where the contract terminates at time $T$ with no surrender charges.

We assume the proceeds from surrender charges are invested in the hedging portfolio. Without surrender charges, it would be optimal to surrender the contract when it is OTM and avoid paying future annual rider fees. Surrender charges act as a disincentive and may make it too costly to surrender, or if surrender is still optimal, they provide the insurer with compensation for the loss of future fees. For GMDB riders, (Milevsky and Salisbury, 2001) argue "when option premiums are paid by instalments - even in the presence of complete mortality and financial markets - the ability to 'lapse' de facto creates an incomplete market". In our view, the surrender charges complete the market and make the guarantees hedgeable. In fact, in the case of a binomial model for the product without mortality we prove in (Hyndman and Wenger, 2013b) that the product can be perfectly hedged with fee income and surrender charges. 
We also explore pricing, hedging errors, and diversification limits in a binomial model with mortality risk in (Hyndman and Wenger, 2013a).

The pricing task, viewed from the policyholder's perspective, becomes an optimal stopping problem. The contract value process for the VA plus GMWB is

$$
V_{t}:=\sup _{\eta \in \mathbb{L}_{t}} V_{t}^{\eta}
$$

where

$$
V_{t}^{\eta}=E_{\mathbb{Q}}\left[G \bar{a} \overline{\eta-t}\left|e^{-r(\eta-t)} W_{\eta}\left(1-k_{\eta}\right)\right| \mathcal{F}_{t}\right]
$$

and $\mathbb{L}_{t}$ is the set of $\mathbb{F}$-adapted stopping times taking values in $[t, T]$. Observe that it is sufficient to consider the set $\mathbb{L}_{t, \bar{\tau}_{t}} \subset \mathbb{L}_{t}$, where $\mathbb{L}_{t, \bar{\tau}_{t}}$ contains all $\mathbb{F}$-adapted stopping times taking values in $\left[t, \bar{\tau}_{t}\right) \cup\{T\}$, and $\bar{\tau}_{t}$ is the trigger time assuming no lapses. That is, if the rider is triggered without prior surrender then due to product design the future guaranteed payments can not be immediately withdrawn and optimal surrender will naturally occur at maturity time $T$.

By the Markov property of $W_{t}$ we have $V_{t}=v\left(t, W_{t}\right) \mathbb{Q}$-a.s. for all $t \in$ $[0, T]$, where $v:[0, T] \times \mathbb{R}_{+} \mapsto \mathbb{R}_{+}$is given by

$$
v(t, x)=\sup _{\eta \in \mathbb{L}_{t, \bar{\tau}_{t}^{x, t}}} E_{\mathbb{Q}}\left[G \overline{\mathrm{a}} \overline{\eta-t}+e^{-r(\eta-t)} W_{\eta}^{x, t}\left(1-k_{\eta}\right)\right] .
$$

Suppose that $k_{0}=0$ and let $\hat{\alpha}:=\inf \left\{\alpha ; V_{0}(P, \alpha, g)=P\right\}$. Then for all $\alpha \geq \hat{\alpha}$ we have $V_{0}(P, \alpha, g)=P$, but there will be no buyers as it is optimal to surrender immediately. Insurers will not charge $\alpha<\hat{\alpha}$ because $V_{0}(P, \alpha, g)>P$. When lapses are permitted but no surrender charges are imposed, there is no unique $\alpha^{\star}$ and the product is not marketable. To preclude this trivial case, we impose the condition that $k_{0}>0$.

The insurer's value process for the rider, analogous to (10), is given by

$$
U_{t}:=\sup _{\eta \in \mathbb{L}_{t, \bar{\tau}_{t}}} U_{t}^{\eta}
$$

where

$$
U_{t}^{\eta}=E_{\mathbb{Q}}\left[G e^{-r\left(\bar{\tau}_{t}-t\right)} \mathbf{1}_{\{\eta=T\}} \overline{\mathrm{a}} \overline{T-\bar{\tau}_{t}}\left|-\int_{t}^{\eta} \alpha e^{-r(s-t)} W_{s} d s-e^{-r(\eta-t)} W_{\eta} k_{\eta}\right| \mathcal{F}_{t}\right] .
$$

We introduce a value process for the option to surrender and denote it by $L=\left\{L_{t} ; 0 \leq t \leq T\right\}$. Let $U_{t}^{N L}$ be the rider value given by (10) in the no-lapse model. Then we define $L_{t}:=U_{t}-U_{t}^{N L} \geq 0$ for all $t \in[0, T]$. It follows that

$$
L_{t}=\sup _{\eta \in \mathbb{L}_{t, \bar{\tau}_{t}}} L_{t}^{\eta},
$$

where

$$
L_{t}^{\eta}=E_{\mathbb{Q}}\left[\int_{\eta}^{T} \alpha e^{-r(s-t)} W_{s} d s-G e^{-r\left(\bar{\tau}_{t}-t\right)} \mathbf{1}_{\left\{\eta<\bar{\tau}_{t}\right\}} \overline{\bar{a}} \frac{\overline{T-\bar{\tau}_{t}} \mid}{-}-k_{\eta} W_{\eta} e^{-r(\eta-t)} \mid \mathcal{F}_{t}\right] .
$$


This formulation is quite intuitive. For a fixed surrender strategy, the surrender benefit is the expected value of the fees avoided by early surrender, less any future benefit payments missed if surrender occurs prior to a trigger time, and less the surrender charge paid at the time of surrender. It is natural that the insured seeks to optimize this surrender benefit. The Markovian representations for $U$ and $L$ are obvious and are omitted.

Proposition 6 formalized the precise relationship between $\left\{U_{t}\right\}$ and $\left\{V_{t}\right\}$ in the no-lapse model. The next theorem generalizes that relationship to the current model and is an extension of a result proved by (Peng et al., 2012) in the case where no lapses are permitted. (Peng et al., 2012) considers a model with stochastic interest rates. In contrast, we suppose interest rates are constant but generalize (Peng et al., 2012, Eqn. (2.16)) to all times $t \in[0, T]$ and allow for lapses.

Theorem 7. Let $V_{t}, U_{t}^{N L}, L_{t}, U_{t}$ be defined by (12), (10), (15) and (14) respectively. Then for all $\alpha \geq 0$ and for all $t \in[0, T]$, we have

$$
V_{t}=W_{t}+U_{t}^{N L}+L_{t}, \quad \mathbb{Q} \text { a.s. },
$$

or, equivalently,

$$
V_{t}=W_{t}+U_{t}, \quad \mathbb{Q} \text { a.s. }
$$

Proof. Fix $t \in[0, T]$. Applying the product rule to $\left(e^{-r(s-t)} W_{s}\right)$ for any $s \in$ $[t, T]$,

$$
\begin{aligned}
d\left(e^{-r(s-t)} W_{s}\right) & =-r e^{-r(s-t)} W_{s} d s+e^{-r(s-t)} d W_{s} \\
& =-r e^{-r(s-t)} W_{s} d s+e^{-r(s-t)}\left[(r-\alpha) W_{s} d s+\sigma W_{s} d B_{s}-G d s\right] \\
& =-\alpha e^{-r(s-t)} W_{s} d s+e^{-r(s-t)} \sigma W_{s} d B_{s}-e^{-r(s-t)} G d s
\end{aligned}
$$

Fix $\eta \in \mathbb{L}_{t, \bar{\tau}_{t}}$. Integrating over the interval $\left[t, \eta \wedge \bar{\tau}_{t}\right]$, and observing that $W_{s \wedge \bar{\tau}_{t}}=W_{s}$ for all $s \in[t, T]$, we obtain

$$
e^{-r(\eta-t)} W_{\eta}-W_{t}=-\int_{t}^{\eta} \alpha W_{s} e^{-r(s-t)} d s-G \overline{\mathrm{a}} \overline{\bar{\eta} \bar{\tau}_{t}-t \mid}+\int_{t}^{\eta} e^{-r(s-t)} \sigma W_{s} d B_{s} .
$$

Note that $G \overline{\mathrm{a}} \overline{\eta-t \mid}=G \overline{\mathrm{a}} \overline{\eta \wedge \bar{\tau}_{t}-t \mid}+G e^{-r\left(\overline{\bar{t}}_{t}-t\right)} \overline{\mathrm{a}} \overline{\eta \vee \bar{\tau}_{t}-\bar{\tau}_{t} \mid}$. Having fixed $\eta \in \mathbb{L}_{t, \bar{\tau}_{t}}$ we have $\overline{\mathrm{a}} \overline{\eta \vee \bar{\tau}_{t}-\bar{\tau}_{t} \mid}=\mathbf{1}_{\{\eta=T\}} \overline{\mathrm{a}} \overline{T-\bar{\tau}_{t} \mid}$. Then

$$
\begin{aligned}
& e^{-r(\eta-t)} W_{\eta}+G \overline{\mathrm{a}} \overline{\eta-t \mid}= \\
& W_{t}+G e^{-r\left(\bar{\tau}_{t}-t\right)} \mathbf{1}_{\{\eta=T\}} \overline{\mathrm{a}} \frac{\overline{T-\bar{\tau}_{t}}}{-}-\int_{t}^{\eta} \alpha W_{s} e^{-r(s-t)} d s+\int_{t}^{\eta} e^{-r(s-t)} \sigma W_{s} d B_{s} .
\end{aligned}
$$

We have, from equation (5), that

$$
E_{\mathbb{Q}}\left[\int_{u}^{v}\left(W_{s}\right)^{2} d s\right]<E_{\mathbb{Q}}\left[\int_{u}^{v} P^{2} e^{2\left(r-\alpha-0.5 \sigma^{2}\right) s+2 \sigma B_{s}} d s\right]<\infty,
$$


thus by a standard result the above Itô integral term is a martingale (see (Øksendal, 2003, Corollary 3.2.6)) and $E_{\mathbb{Q}}\left[\int_{t}^{\eta} e^{-r(s-t)} \sigma W_{s} d B_{s} \mid \mathcal{F}_{t}\right]=0$. Subtracting $e^{-r(\eta-t)} W_{\eta} k_{\eta}$ from both sides and taking conditional expectations w.r.t. $\mathcal{F}_{t}$, we obtain

$$
V_{t}^{\eta}=W_{t}+U_{t}^{\eta}
$$

Since $\eta$ was arbitrary, taking the supremum gives

$$
V_{t}=W_{t}+U_{t}
$$

Corollary 8. For any $\alpha \geq 0$,

$$
L_{t}=\sup _{\eta \in \mathbb{L}_{t, \bar{\tau}_{t}}} E_{\mathbb{Q}}\left[e^{-r(\eta-t)} W_{\eta}\left(1-k_{\eta}\right)-e^{-r(T-t)} W_{T}-G e^{-r \eta} \overline{\mathrm{a}} \overline{T-\eta} \mid \mathcal{F}_{t}\right] .
$$

Proof. Proposition 6 and Theorem 7 imply $L_{t}=V_{t}-V_{t}^{N L}$ from which (18) is obtained.

Remark. For $\alpha^{\star}$, such that $V_{0}=P$, we have that $U_{0}\left(\alpha^{\star}\right)=0$ and $L_{0}\left(\alpha^{\star}\right)=$ $-U_{0}^{N L}\left(\alpha^{\star}\right)$. Equation (18) is interpreted as the insured selecting the surrender time to maximize the trade-off between receiving the account value (less surrender charges) today, rather than at maturity, and foregoing the rights to any future withdrawals.

\section{Conclusions}

In this paper we have considered the valuation problem of a variable annuity with a guaranteed minimum withdrawal benefit rider from the perspective of both the policy holder and the insurer. The focus and main contributions of this paper are the financial aspects of the variable annuity and GMWB rider. We define the fair rider fee as the rate which equates the risk-neutral expectation of all future benefits to the insured to the initial premium. The first contribution of the paper is a proof of the existence and uniqueness of the fair fee. The second contribution of the paper is an extension of the decomposition results of (Peng et al., 2012) to include lapses. We decompose the value of the contract into the account value, a component expressing the value of the guarantee in the no-lapse case, and a component expressing the value of the option to lapse.

The valuation perspectives and decompositions are expressed in terms of optimal stopping problems which require a numerical implementation technique to value the contract. We referred earlier to a subset of articles which focus on various approaches. Rather than directly applying a numerical approach to the continuous time results presented in this paper, which are of independent interest, we focus on the fundamental theory here and place an emphasis on numerical implementation in our subsequent works. In (Hyndman and Wenger, 2013b) we construct a complete binomial framework for the contract recovering similar valuation perspectives and decompositions to those presented in this paper, and include hedging results for the GMWB rider as well as numerical implementation techniques. In particular, in (Hyndman and Wenger, 
2013b), we obtain numerical results based on the binomial model which can be viewed as an approximation to the model presented in this paper and which are in accordance with numerical results obtained using Monte-Carlo methods by (Kolkiewicz and Liu, 2012) and (Liu, 2010). Further, while we disregard mortality in this paper we extend the binomial modelling framework to include mortality and obtain numerical results demonstrating the limits of hedging and diversification of mortality risk in (Hyndman and Wenger, 2013a).

Acknowledgements This research was supported by the Natural Sciences and Engineering Research Council (NSERC) of Canada and the Fonds de recherche du Québec - Nature et technologies (FQRNT). The authors thank the editor and anonymous referee for valuable comments which improved the paper.

\section{Appendix A. Additional Results and Proofs}

This appendix is devoted to the proofs of Lemma 4 and Theorem 5 . The following two lemmas are required to prove Lemma 4 .

Lemma A1. For any $T, a, k>0$ we have $\mathbb{Q}\left(\int_{0}^{T} e^{-a B_{s}} d s<k\right)>0$, where $B_{s}$ is a standard $\mathbb{Q}$-Brownian motion process.

Proof. 1 Let $u=\min \left(\frac{k}{2 e^{a}}, T\right)$. Write $\int_{0}^{T} e^{-a B_{s}} d s=\int_{0}^{u} e^{-a B_{s}} d s+\int_{u}^{T} e^{-a B_{s}} d s$. We consider the two cases $u=T$ and $u<T$ separately by conditioning on the events $A=\left\{B_{s}>-1 ; \forall s \in[0, u]\right\}$ and $C=\left\{B_{s}>M ; \forall s \in[u, T]\right\}$, where $M$ satisfies $e^{-a M}=\frac{k}{2(T-u)}$.

(i) $u=T$ :

Conditioning on the event $A$ we find that

$$
\mathbb{Q}\left(\int_{0}^{T} e^{-a B_{s}} d s<k\right) \geq \mathbb{Q}\left(\int_{0}^{T} e^{-a B_{s}} d s<k \mid A\right) \mathbb{Q}(A)=\mathbb{Q}(A)
$$

since $A$ implies $\int_{0}^{T} e^{-a B_{s}} d s<T e^{a} \leq \frac{k}{2}$ and $\mathbb{Q}\left(\int_{0}^{T} e^{-a B_{s}} d s<k \mid A\right)=1$. By (Borodin and Salminen, 2002, formula 1.1.2.4)

$$
\mathbb{Q}_{x}\left(\inf _{0 \leq s \leq t} \tilde{B}_{s}>y\right)=2 \Phi\left(\frac{x-y}{\sqrt{t}}\right)-1, \quad y \leq x,
$$

where $\Phi$ is the cdf of the standard normal distribution and $\tilde{B}_{t}$ is a Brownian motion with $\tilde{B}_{0}=x$ a.s. under $\mathbb{Q}_{x}$. Hence, equation (A.1), with $x=0 ; y=-1$; and $t=T$, implies that $\mathbb{Q}(A)>0$. That is, $\mathbb{Q}\left(\int_{0}^{T} e^{-a B_{s}} d s<k\right)>0$ as desired.

\footnotetext{
${ }^{1}$ The authors thank Dr. Anthony Quas, University of Victoria, for helpful discussion concerning the proof of Lemma A1
} 
(ii) $u<T$ :

Conditioning on the events $A$ and $C$ we find that

$$
\begin{aligned}
\mathbb{Q}\left(\int_{0}^{T} e^{-a B_{s}} d s<k\right) & \geq \mathbb{Q}\left(\int_{0}^{u} e^{-a B_{s}} d s+\int_{u}^{T} e^{-a B_{s}} d s<k \mid A \cap C\right) \mathbb{Q}(A \cap C) \\
& =\mathbb{Q}(A \cap C)
\end{aligned}
$$

since $A$ implies $\int_{0}^{u} e^{-a B_{s}} d s<u e^{a}=\frac{k}{2}$ and $C$ implies $\int_{u}^{T} e^{-a B_{s}} d s<(T-$ $u) e^{-a M}=\frac{k}{2}$. Similar to the first case we have $\mathbb{Q}(A)>0$ by equation (A.1). To see $\mathbb{Q}(C \mid A)>0$, we introduce $D=\left\{B_{u}>M+\epsilon\right\}$ where $\epsilon>0$ and $B_{0}=0$. Then $\mathbb{Q}(D)>0$. We could use reflection-type arguments to show that $\mathbb{Q}(A \mid D)>0$ but we refer to a more complete result in (Jeanblanc et al., 2009, Proposition 4.3.5.3) on the maximum of a general Brownian bridge. Conditioning on $D$ and taking expectations w.r.t. $B_{u}$, that result can be used to derive an explicit expression for $\mathbb{Q}(A \mid D)$. Therefore $\mathbb{Q}(D \mid A)>0$.

Finally, equation (A.1), with $x=M+\epsilon ; y=M$; and $t=T-u$, implies $\mathbb{Q}(C \mid A \cap D)>0$. Thus

$$
\mathbb{Q}(A \cap C)=\mathbb{Q}(A) \mathbb{Q}(C \mid A) \geq \mathbb{Q}(A) \mathbb{Q}(C \mid A \cap D) \mathbb{Q}(D \mid A)>0 .
$$

Lemma A2. For any fee rate $\alpha$ and guaranteed withdrawal rate $g$ there is a positive probability that the contract matures with a positive account value. That is,

$$
\mathbb{Q}\left(W_{T}^{P, 0}>0\right)>0
$$

for all $P>0, g>0$, and $\alpha \geq 0$, where $W_{T}^{P, 0}$ is given by (4).

Proof. Note that $W_{T}^{P, 0}>0$ if and only if

$$
\frac{P}{G}>\int_{0}^{T} e^{-\left(r-\alpha-0.5 \sigma^{2}\right) s-\sigma B_{S}} d s .
$$

By bounding and removing the deterministic portion from the integrand, we have

$$
\frac{P}{G}>\int_{0}^{T} e^{-\left(r-\alpha-0.5 \sigma^{2}\right) s-\sigma B_{S}} d s
$$

if

$$
\frac{P}{G} c^{-1}>\int_{0}^{T} e^{-\sigma B_{s}} d s
$$

where

$$
c= \begin{cases}e^{-\left(r-\alpha-0.5 \sigma^{2}\right) T} & \text { if }\left(r-\alpha-0.5 \sigma^{2}\right)<0, \\ 1 & \text { otherwise. }\end{cases}
$$

The desired conclusion follows from that fact that $\mathbb{Q}\left(\int_{0}^{T} e^{-a B_{s}} d s<k\right)>0$ for all $T, a, k>0$ of Lemma A1. 
Proof of Lemma 4. We fix $P$ and $g$ and omit them from the notation. A monotonicity result is obtained by applying a comparison result for SDEs from Karatzas and Shreve, 1991, Proposition 2.18). Since $\alpha$ appears as a negative drift term in the SDE for $W_{t}$ in (3), we have $W_{t}\left(\alpha_{1}\right) \geq W_{t}\left(\alpha_{2}\right)$ a.s. for all $t \in[0, T]$ and for all $0 \leq \alpha_{1}<\alpha_{2}$. Thus $E_{\mathbb{Q}}\left[W_{T}\left(\alpha_{1}\right)\right] \geq E_{\mathbb{Q}}\left[W_{T}\left(\alpha_{2}\right)\right]$ which implies $V_{0}\left(\alpha_{1}\right) \geq V_{0}\left(\alpha_{2}\right)$.

To prove the strictly decreasing property of $V_{0}(P, \alpha, g)$ note from Lemma A2 that $\mathbb{Q}\left(A^{\alpha}\right)>0$ for all $\alpha \geq 0$ where $A^{\alpha}:=\left\{W_{T}(\alpha)>0\right\}$. On the event $A^{\alpha}$ we have

$$
W_{T}(\alpha)=e^{\left(r-\alpha-0.5 \sigma^{2}\right) T+\sigma B_{T}} \times\left(P-G \int_{0}^{T} e^{-\left(r-\alpha-0.5 \sigma^{2}\right) s-\sigma B_{s}} d s\right) .
$$

Let $0 \leq \alpha_{1}<\alpha_{2}=\alpha_{1}+h$, where $h$ takes an arbitrary positive value. Restricted to the set $A^{\alpha_{1}+h}$, we obtain

$$
W_{T}\left(\alpha_{1}+h\right) \leq e^{-h T} W_{T}\left(\alpha_{1}\right)<W_{T}\left(\alpha_{1}\right)
$$

implying that $A^{\alpha_{1}} \supseteq A^{\alpha_{1}+h}$. It follows that

$$
\begin{aligned}
V_{0}\left(\alpha_{1}+h\right) & =G \bar{a}_{T \mid}+E_{Q}\left(e^{-r T} W_{T}\left(\alpha_{1}+h\right) \mathbf{1}_{\left\{A^{\alpha_{1}+h}\right\}}\right) \\
& <G \bar{a}_{T}+E_{Q}\left(e^{-r T} W_{T}\left(\alpha_{1}\right) \mathbf{1}_{\left\{A^{\alpha_{1}+h}\right\}}\right) \\
& \leq V_{0}\left(\alpha_{1}\right) .
\end{aligned}
$$

To prove continuity fix $\alpha \geq 0$. Let $h>0$ and denote

$$
X_{T}^{h}:=e^{\sigma B_{T}} \max \left(0, P-G \int_{0}^{T} e^{-\left(r-\alpha-h-\frac{1}{2} \sigma^{2}\right) s-\sigma B_{s}} d s\right) .
$$

From (44),

$$
E_{Q}\left(W_{T}(\alpha+h)\right)=e^{\left(r-\alpha-h-\frac{1}{2} \sigma^{2}\right) T} E_{Q}\left(X_{T}^{h}\right) .
$$

Then $X_{T}^{h} \geq 0$ for all $h \geq 0$, and $X_{T}^{h} \uparrow$ a.s. as $h \downarrow 0$. Applying the Monotone Convergence theorem and by the continuity of the max function,

$$
\lim _{h \downarrow 0} E_{\mathbb{Q}}\left(X_{T}^{h}\right)=E_{\mathbb{Q}}\left(X_{T}^{h=0}\right) .
$$

The Dominated Convergence theorem was used to interchange the limit and the path-wise Lebesgue-Stieltjes integral. Therefore $\lim _{h \downarrow 0} E_{\mathbb{Q}}\left(W_{T}(\alpha+h)\right)=$ $E_{\mathbb{Q}}\left(W_{T}(\alpha)\right)$.

If $\alpha>0$, then let $h<0$ and $\lim _{h \uparrow 0} E_{\mathbb{Q}}\left(W_{T}(\alpha+h)\right)=E_{\mathbb{Q}}\left(W_{T}(\alpha)\right)$ is obtained using similar arguments. The Monotone Convergence theorem no longer applies; instead the Dominated Convergence theorem justifies interchanging the expectation and limit since $X_{T}^{h} \leq P e^{\sigma B_{T}}$ and $E_{\mathbb{Q}}\left(e^{\sigma B_{T}}\right)=e^{0.5 \sigma^{2} T}<\infty$. Therefore the continuity of $V_{0}$ follows from (6) . 
Proof of Theorem [5. The existence of $\alpha^{\star}$ is obtained by showing that both $V_{0}(P, 0, g) \geq P$ and $\lim _{\alpha \rightarrow \infty} V_{0}(P, \alpha, g)<P$ and applying the continuity result from Lemma 4

When $\alpha=0$, the guarantee is offered at no charge and it is obvious that $V_{0} \geq P$. More formally, setting $\alpha=0$ we have from (4)

$$
W_{T} \geq\left[P e^{\left(r-0.5 \sigma^{2}\right) T+\sigma B_{T}}-G \int_{0}^{T} e^{\left(r-0.5 \sigma^{2}\right)(T-s)+\sigma\left(B_{T}-B_{s}\right)} d s\right],
$$

and since $E_{\mathbb{Q}}\left[e^{-0.5 \sigma^{2} t+\sigma B_{t}}\right]=1$, we obtain from (6) that

$$
V_{0}(P, 0, g) \geq P+E_{\mathbb{Q}}\left[\int_{0}^{T} e^{-r s} G\left(1-e^{-\left(0.5 \sigma^{2}\right)(T-s)+\sigma\left(B_{T}-B_{s}\right)}\right) d s\right]=P,
$$

where the expectation on the right evaluates to zero by Fubini's theorem.

As $\alpha \rightarrow \infty$, it becomes certain that the embedded GMWB option will be exercised and thus $V_{0}=G \bar{a}_{T \text {. }}$. More formally, for $\alpha>0$ we have

$$
0 \leq W_{T}(\alpha) \leq P e^{-\alpha T} e^{\left(r-0.5 \sigma^{2}\right) T+\sigma B_{T}} \leq P e^{\left(r-0.5 \sigma^{2}\right) T+\sigma B_{T}}
$$

a.s., and $E_{\mathbb{Q}}\left[P e^{\left(r-0.5 \sigma^{2}\right) T+\sigma B_{T}}\right]=P e^{r T}<\infty$. The property $B_{T}<\infty$ a.s. combined with (A.2) gives $\lim _{\alpha \rightarrow \infty} W_{T}(\alpha)=0$ a.s. Applying the Dominating Convergence theorem,

$$
\lim _{\alpha \rightarrow \infty} V_{0}(P, \alpha, g)=G \int_{0}^{T} e^{-r s} d s<G T=P,
$$

for $r>0$.

The uniqueness of the solution follows directly from the strictly decreasing property for $V_{0}(P, \alpha, g)$ from Lemma 4 .

\section{References}

Bacinello, A.R., Millossovich, P., Olivieri, A., Pitacco, E., 2011. Variable annuities: A unifying valuation approach. Insurance: Mathematics and Economics 49, 285-297.

Bauer, D., Kling, A., Russ, J., 2008. A universal pricing framework for guaranteed minimum benefits in variable annuities. ASTIN Bulletin 38, 621-651.

Borodin, A.N., Salminen, P., 2002. Handbook of Brownian Motion: Facts and Formulae. Birkhäuser, Basel, Boston, Berlin. 2nd edition.

Chen, Z., Forsyth, P.A., 2008. A numerical scheme for the impulse control formulation for pricing variable annuities with a guaranteed minimum withdrawal benefit (GMWB). Numerische Mathematik 109, 535-569. 
Dai, M., Kwok, Y.K., Zong, J., 2008. Guaranteed minimum withdrawal benefit in variable annuities. Mathematical Finance 18, 595-611.

Hyndman, C., Wenger, M., 2013a. Diversification of mortality risk in GMWB rider pricing and hedging. Working paper.

Hyndman, C., Wenger, M., 2013b. Pricing and hedging GMWB riders in a binomial framework. Working paper.

Jeanblanc, M., Yor, M., Chesney, M., 2009. Mathematical Methods for Financial Markets. Springer Finance, London.

Karatzas, I., Shreve, S.E., 1991. Brownian Motion and Stochastic Calculus. Springer-Verlag, New York. 2nd edition.

Kolkiewicz, A., Liu, Y., 2012. Semi-static hedging for GMWB in variable annuities. North American Actuarial Journal 16, 112-140.

Levy, E., 1992. Pricing European average rate currency options. Journal of International Money and Finance 11, 474-491.

Liu, Y., 2010. Pricing and Hedging the Guaranteed Minimum Withdrawal Benefits in Variable Annuities. Ph.D. thesis. University of Waterloo.

Milevsky, M.A., Salisbury, T.S., 2001. The real option to lapse a variable annuity: Can surrender charges complete the market? Conference Proceedings of the 11th Annual International AFIR Colloquium v2, 537-561.

Milevsky, M.A., Salisbury, T.S., 2006. Financial valuation of guaranteed minimum withdrawal benefits. Insurance: Mathematics and Economics 38, 21-38.

Øksendal, B.K., 2003. Stochastic Differential Equations: An Introduction with Applications. Springer Verlag, Berlin, Heidelberg, and New York. 6th edition.

Peng, J., Leung, K.S., Kwok, Y.K., 2012. Pricing guaranteed minimum withdrawal benefits under stochastic interest rates. Quantitative Finance 12, 933941. 